\title{
À propos d'un passage des Lettres Familières de Pétrarque (Ep. III, 18) : de la bibliographie comme discours du contenu et de la méthode
}

\author{
Laurence Pradelle \\ Université de Limoges
}

$\mathrm{Si}$, selon une définition imparfaite mais sur laquelle il est possible de s'accorder dans un premier temps, on entend par bibliographie un récapitulatif aussi exhaustif que possible des sources concernant un sujet donné, il semblera a priori surprenant de faire entrer dans l'histoire de la bibliographie une épître de Pétrarque, perdue au milieu du troisième livre des vingt-quatre qui composent les Lettres Familières ${ }^{1}$.

1 Sur la genèse et la portée de cette correspondance, voir Pétrarque. 2002, vol. I, introduction, p. XIII-LXXXV (références ultérieures : Dotti, 2002). 
Pour ce qui est du livre, tout d'abord, il paraît être d'une importance assez relative par rapport à ceux qui l'entourent, si bien que la dix-huitième lettre, qui n'a sa place ni en ouverture ni en conclusion, ne ferait qu'appartenir modestement à un livre de transition ${ }^{2}$. Ensuite, pour ce qui est de la lettre ellemême, qui est une requête adressée à un ami, Giovanni Anchiseo, censé lui trouver des livres, elle se présente avant tout comme un éloge de la littérature et l'humaniste semble surtout procéder par association d'idées: les auteurs et les œuvres cités ne paraissent pas toujours avoir de liens entre eux, si ce n'est pour le seul Pétrarque ; ce dernier intercale auteurs majeurs et mineurs sans volonté de hiérarchisation, mais en passant des uns aux autres en fonction de liens subjectifs que lui-même aurait établis et que, d'une certaine manière, lui seul connaît, sans les rapporter à un objet précis. La première impression du lecteur est qu'ils sont jetés là au hasard et mis en relation les uns avec les autres de manière arbitraire, peut-être pour donner une impression de multiplicité. À la rigueur, on pourrait penser que Pétrarque nous livre, à ce moment-là de sa correspondance, un inventaire de sa bibliothèque à l'époque de la lettre, qu'il faut situer après $1345^{3}$. Mais il suffit de faire la comparaison avec la dernière page du Parisinus latinus 2201, qui décrit un état antérieur de sa bibliothèque (1337), pour écarter cette hypothèse : les livres cités dans la liste sont loin de

2 Dotti a montré que les livres I et II, programmatiques, servaient d'introduction à l'œuvre des Familières en évoquant les grands thèmes de la nouvelle littérature humaniste ainsi que les vicissitudes intérieures rencontrées par Pétrarque. Le livre IV porte sur le couronnement au Capitole. Le livre III est plus difficile à cerner, ne comportant pas d'unité aussi marquée.

Voir Dotti, 2002, vol. I, p. 233-234.

3 Il y est question des Lettres à Atticus, découvertes en 1345. Voir Dotti, 2002, vol. I, p. 458. 
recouvrir l'ensemble des livres de sa bibliothèque, quelquesuns ne font pas partie de celle-ci et, surtout, la disposition ne présente aucun rapport ${ }^{4}$. La liste de la lettre III, 18 n'est pas seulement - un relevé de la bibliothèque de Pétrarque.

Dès lors, pourquoi lire cette lettre comme une possible bibliographie et non plutôt comme un simple épanchement du poète, faisant part à son ami de sa passion pour les livres ? Il se trouve que Pétrarque nous y invite discrètement en indiquant deux sortes de rapports possibles entre les œuvres (ou les auteurs) présentes dans la liste : le desiderium et l'auctoritas. En effet, juste avant son énumération, il affirme : «Non seulement chacun d'eux pénètre dans l'esprit de ceux qui les lisent, mais il leur suggère le nom d'autres livres et de l'un à l'autre se nourrit le désir $»^{5}$ (Pétrarque, Fam. III, 18, 3). Quant à l'auctoritas, elle se révèle à travers de nombreux termes tels que commendauit, admonuit, testis illustrior, etc. Pétrarque tient visiblement à l'alliance des deux, suggérant que la dimension subjective du choix d'auteurs n'est pas séparable d'une dimension objective, visant à l'universalité. Cette double exigence, si elle nous achemine déjà vers l'idée de bibliographie, serait peut-être insuffisante pour étudier la lettre dans le contexte de la bibliographie, si ne venaient s'ajouter deux autres raisons, l'une extrinsèque à la lettre, l'autre intrinsèque.

La première est suggérée par les gloses que Pétrarque a laissées de sa main (Nolhac, 1907, vol. I, p. 123-161). Décrivant notamment l'un des manuscrits fétiches du poète florentin,

\footnotetext{
4 Nolhac, 2004, vol. II, p. 293-296, excursus VII : « le catalogue de la première bibliothèque de Vaucluse » (références ultérieures : Nolhac, 1907).

${ }^{5}$ [...] neque solum se se lectoribus quisque suis insinuat, sed et aliorum nomen ingerit et alter alterius desiderium facit.
} 
celui qui est aujourd'hui connu sous le nom de «Virgile de l'Ambrosienne», P. de Nolhac en retrace l'histoire puis s'intéresse au très grand nombre de notes marginales de Pétrarque et à ses commentaires sur les œuvres du poète latin. Il ressort de ce passage que les références données par Pétrarque dans les marges de son Virgile sont de deux ordres : on y trouve celles qui concernent classiquement le texte virgilien, celles que l'on rangerait sans hésitation sous la rubrique des notes correspondant à nos actuelles « notes de bas de page » et dans lesquelles figurent soit des précisions sur le texte, visant à l'éclaircir et à le commenter, soit une mise en rapport du texte présent avec d'autres textes (les implications chez d'autres auteurs, ou bien les sources) : il s'agit là d'une démarche bibliographique de base déjà répandue avant notre auteur; et il y a les autres, celles qui sont « sans rapport direct avec le texte » et qui, en somme, concernent moins Virgile que Pétrarque, au sens où elles laissent poindre davantage la méthode de travail personnelle de l'auteur. Il se pourrait que celles-ci appartiennent à une démarche d'un autre ordre. Sur ce point, P. de Nolhac hésite : il commence par dire qu'il ne s'agit pas de pédantisme, c'est-à-dire de démonstration voyante, arbitraire, personnelle et, au fond, gratuite de son érudition, mais plutôt d'une sorte de « répertoire, où le travailleur vient chercher ses références »; puis il glisse vers l'idée de « florilège personnel » qui, en retenant les passages les plus élégants, semble avoir pour but unique de faire plaisir aux amis. Dans ce cas, on en reviendrait ni plus ni moins à une forme de pédantisme. Qu'il y ait de cela, Pétrarque le reconnaît lui-même dans le Secretum. Enfin, P. de Nolhac parle de «résumé de la bibliothèque" et de "la première ébauche d'un Virgilius illustratus» (1907, vol. I, p. 160-161). 
Quoi qu'il en soit, il semblerait qu'il y ait depuis longtemps, chez Pétrarque, une tendance à rechercher, mais aussi et surtout à classer et ordonner les sources à sa disposition ainsi qu'un fonds de références plus indirectes, dans le but de créer des outils de travail permettant, à lui-même et aux autres, un maniement plus facile des diverses informations, mais aussi une certaine orientation de celles-ci. Cette double attitude serait la conséquence d'un traitement complexe des sources, considérées tantôt du point de vue du contenu, tantôt à partir d'une réflexion plus large sur la manière de les aborder.

Il existe une autre raison. Cette liste d'auteurs, telle qu'elle nous est présentée, laisse perplexe du fait que, à partir $\mathrm{du}$ moment où l'on se refuse à n'y voir qu'un égrainement subjectif d'auteurs, on ne sait toujours pas comment la caractériser : elle ne relève en effet ni du canon d'auteurs, dont on trouve un modèle bien plus tard dans les Familières (Fam. XVIII, 1-4), ni de la doxographie, dont on a, par ailleurs, un bel exemple dans la première lettre de ce même troisième livre, où est posée avec acuité la question de l'utilité d'une recherche systématique des sources. En effet, cherchant à renseigner son ami Tommaso Caloiro sur l'emplacement de l'île de Thulé, Pétrarque se lance dans un recensement exhaustif des informations circulant sur le sujet depuis l'Antiquité, pour en arriver au fait que personne ne sait où elle se trouve : l'île, qui ne lui « sembla pas moins cachée que la vérité »6 (III, 1, 13), en devient alors pour ainsi dire la métaphore. L'humaniste se livre ici à une critique virulente d'une certaine pratique érudite qui consiste à faire appel à toutes les autorités existantes sans pour autant chercher vraiment à obtenir de réponse ni de révélation,

${ }^{6}$ Ut michi quidem nichilo uideatur occultior insula ipsa quam ueritas. 
parce qu'elle se trompe d'objet. Les sources, aussi complètes soient-elles, ne nous mènent pas nécessairement à la vérité tant qu'on les utilise " pour sonder les recoins et les secrets de la nature », et non pour se « connaître soi-même ${ }^{7}$ (Pétrarque, III, $1,14)$. Et l'on perçoit d'emblée que l'un des problèmes posés par ce livre (qui, dès lors, acquiert une autre dimension) est énoncé dès la première phrase de cette première lettre : "Qui s'aventure aux confins du monde des Anciens, d'accès difficile certes mais pleins d'agrément pour qui y parvient, doit fouler en pensée un chemin semé d'obstacle $»^{8}$ (Pétrarque, III, 1, 1). L'objet du troisième livre des Familières serait peut-être en rapport avec l'exigence de trouver son chemin dans le labyrinthe de la tradition, dans ce dédale qu'est la littérature des Anciens : autant il ne faut pas renoncer à la connaissance et gaspiller son temps dans la recherche de biens matériels (c'est l'objet de la lettre suivante, Fam. III, 2), autant un parcours sans méthode est voué à l'échec, parce qu'une érudition sans direction n'éclaire pas le chemin. Et c'est précisément ce à quoi s'emploie le livre III : découvrir les conditions de la véritable connaissance, de la véritable voie pour s'approprier les Anciens ${ }^{9}$.

\footnotetext{
7 Michi quidem si hec nature latibula rimari et abdita nosse negatum fuerit, me ipsum nosse sufficiet.

8Perambulanti ueterum confinia, accessu quidem aspera sed amena cum perueneris, creber scrupulus ingenii pede calcandus est.

9 On pourrait ajouter une troisième raison justifiant de relier notre lettre III, 18 à la bibliographie : par l'intermédiaire de Pline l'Ancien et de son Histoire Naturelle, qui est l'une des sources importantes des Familières, Pétrarque était rompu à une certaine méthode bibliographique. Sur l'admiration que l'humaniste portait à Pline, voir Nolhac, 1907, vol. II, p. 68-83. Pétrarque entra en possession d'un Pline, le Parisinus 6802 (datant du XIIIe siècle) en juillet 1350 à Mantoue.
} 
L'hypothèse émise pour la présente étude a donc été la suivante : conformément à la démarche de Pétrarque mise en lumière par Pierre de Nolhac à propos du «Virgile de l'Ambrosienne ", conformément aussi à la critique d'une certaine attitude encyclopédique faite par Pétrarque dans la première lettre du livre III, on peut se demander si, derrière la liste apparemment livrée au hasard de l'inspiration proposée dans la lettre 18, il n'est pas possible de repérer des critères de mise en ordre et, à partir de là, de s'interroger sur les rapports éventuels de cet ordre avec le livre III, dans lequel il s'intègre, voire avec les livres précédents. En d'autres termes, quelle est la place de la lettre 18 dans l'économie des trois premiers livres des Familières?

Avant d'en venir précisément à l'analyse de cette lettre, il faut donc commencer par présenter, dans leur contenu et succinctement, les trois livres en question.

\section{Le troisième livre des Familières : interrogations théoriques et réponses pratiques}

Dans les deux premiers livres, Pétrarque expose les grands thèmes humanistes que l'on peut résumer ainsi : quelle attitude adopter face au désir de gloire et à la fuite obsédante du temps? Quelles orientations donner à la nouvelle culture? Le troisième livre reprend ces thèmes en les enrichissant et en ajoute un nouveau : la question de l'engagement politique ${ }^{10}$.

C'est sur la forme et sur l'agencement de ce livre que l'on peut s'arrêter davantage. On y repère en effet des séries

10 Voir Dotti, 2002, p. 3-15, 115-127 et 233-239. 
récurrentes. Par exemple, un premier groupe de lettres (1-10) obéit à une structure tripartite : les deux premières portent sur la question du savoir; les deux suivantes sur l'engagement politique ${ }^{11}$; les six dernières lettres comportent une visée morale : apparemment d'une grande variété de thèmes, elles sont en réalité reliées entre elles par une unité profonde - la lutte contre les trois formes de passions (libido dominandi, libido sciendi, libido sentiendi) - et par un jeu subtil d'échos avec les premières lettres du livre, où, grâce à une mise en abîme, Pétrarque revient sur la triple question du savoir, de l'engagement politique et des passions auxquelles la mort seule, au fond, peut mettre un terme.

Or un deuxième groupe de lettres (11-17) obéit à la même logique tripartite: à une première lettre d'autocélébration du poète ${ }^{12}$, qui définit au sein de la société la place de cette nouvelle figure intellectuelle que Pétrarque appelle de ses vœux, à savoir l'humaniste, fait suite une lettre politique ${ }^{13}$. Juxtaposées, les deux lettres forment un tout faisant écho aux quatre premières lettres du livre, en confrontant une fois de plus vie intellectuelle, plutôt retirée et consacrée au savoir, et vie active, plutôt engagée politiquement. Puis arrivent, comme dans la première partie, des lettres à visée morale, où il est question de dénoncer certains vices de l'époque ${ }^{14}$.

\footnotetext{
11 Pétrarque, Fam. III, 3-4. Ces lettres évoquent le 22 mai 1333 et la rixe entre les Orsini et les Colonna : Pétrarque s'est engagé auprès de ces derniers.

12 Ibid. III, 11. L'auteur y compare la relation que lui-même entretient avec Guido Gonzaga, seigneur de Mantoue, à celle qui unit Virgile et Horace à l'empereur Auguste.

13 Ibid. III, 12.

${ }^{14}$ La lettre III, 17, notamment, d'influence sénéquienne, porte sur le rapport entre réflexion et action et résout, en quelque sorte, le problème du livre III en
} 
À ces deux cycles de lettres succède la lettre 18, objet de notre étude. Pour U. Dotti, «c'est la tesselle qui manquait, ici au seuil des Familiares, à la mosaïque de l'autoportrait de l'humaniste » (p. 238). On peut ajouter, et c'est en cela qu'elle nous intéresse ici, qu'elle marque une césure profonde dans le livre, puisque les quatre lettres restantes apparaissent, nous semble-t-il, comme un excursus ou plutôt comme une sorte d'illustration dans le réel de la théorie déployée dans les dixsept premières lettres et n'obéissent plus à la logique tripartite qui rythmait la première partie.

En effet, les lettres 19-22, à Tosetti, son cher Lélius (1346-47), peuvent être lues comme la narration en quatre temps des progrès spirituels du poète, depuis sa propre soumission aux passions (le désir immodéré d'un canonicat : III, 19), en passant par la mise à l'épreuve imposée par l'ami (la prise de distance par rapport au canonicat: III, 20) et par l'ouverture à l'autre (une nouvelle demande de service, mais pour autrui : III, 21) jusqu'à la libération finale (découverte de l'abnégation: III, 21). Bien des aspects, évoqués sur un plan théorique au cours de la première partie du livre, se retrouvent traités ici sur un plan pratique : la victoire sur les passions, une certaine remise en cause du statut hiérarchique des classes sociales et donc l'amitié possible entre hommes de conditions diverses, pour finir par l'engagement pour autrui de Pétrarque, qui s'efface en tant qu'homme, même aux yeux de son fermier, pour mieux renaître en tant que poète (assurant ainsi la transition avec le livre suivant).

associant, sans plus les opposer, vie contemplative et vie active, cogitare et uiuere. 
Le livre III pourrait donc se résumer ainsi : les lettres 1 à 17 décrivent un cheminement théorique, où se déploient en deux temps (le problème - posé dans les lettres 1-10 - et une esquisse de résolution - dans les lettres 11-17) les questionnements posés par la libido sciendi, la libido dominandi, la libido sentiendi et une certaine manière de les traiter en faisant appel aux auctoritates antiques; la lettre 18 offrirait une récapitulation de ces dernières; les lettres 19 à 22 constitueraient une sorte d'épreuve du réel et donc la preuve par l'expérience de la validité d'une connaissance approfondie des Anciens. Ainsi, la lettre III, 18 arriverait bien à la fin de l'introduction théorique (les formes et les enjeux de la nouvelle culture humaniste) que représentent les trois premiers livres des Familières. À partir du livre IV, le recueil prend une autre tournure, plus autobiographique et historique, en tout cas moins théorique. Pour aller plus loin encore dans le sens de U. Dotti, on peut dire qu'il faut compter le livre III des Familières au nombre des livres d'introduction théorique et terminer ce livre III à la lettre 18, qui acquiert alors un relief incontournable.

Si l'on se concentre maintenant plus spécifiquement sur cette lettre 18, on peut partir d'un présupposé simple: de même que le troisième livre, comme les deux précédents, obéit à une architecture très rigoureuse, de même il est possible de déceler sous l'apparente prolifération arbitraire de titres d'œuvres éparses une véritable classification répondant à chacun des aspects thématiques évoqués. 


\section{La lettre III, 18 ou l'émergence d'un recensement et d'un classement des sources}

Dans un premier temps, il est assez facile de repérer que tous les noms, ou presque, cités dans cette liste d'ouvrages correspondent aux sources sur lesquelles s'est appuyé Pétrarque dans ses trois livres. Mais ce n'est pas le point le plus important, puisque certains auteurs comme Suétone ou Sénèque le Rhéteur n'apparaissent que dans cette lettre III, 18 et que, par ailleurs, certaines sources récurrentes comme Orose ou Justin ne figurent pas dans cette liste. Ce qui paraît plus significatif est que cette liste semble progresser par groupes thématiques. Un premier ensemble concerne, en effet, plutôt la philosophie, d'abord latine puis grecque ; lui succède un groupe où cohabitent pêle-mêle des écrivains chrétiens, des écrivains païens et des historiens; ensuite, les auteurs sont davantage liés à l'éloquence et à la poésie ; enfin, à l'érudition et à la culture encyclopédique. C'est la signification et la cohérence de ces différents groupes que l'on peut interroger et leur relation avec le livre III.

Pour ce qui est du premier groupe, Pétrarque commence par Cicéron, dont il évoque quatre œuvres : les Académiques, les Devoirs, les Tusculanes, le Cato Maior. Chacune de ces œuvres, choisies uniquement parmi les œuvres philosophiques de Cicéron, est mise en rapport avec des œuvres ou des auteurs antiques: les Académiques avec Varron ${ }^{15}$, les Devoirs avec Ennius, les Tusculanes, avec Térence, le Cato Maior, avec Caton (les Origines) et Xénophon (l'Économique).

15 Cicéron et Varron sont souvent présentés de pair par Pétrarque, comme s'ils étaient complémentaires. Voir, par exemple, Pétrarque, Fam. XVIII, 4. 
Deux questions se posent alors: pourquoi, dans cette lettre, Pétrarque s'intéresse-t-il précisément à ces quatre œuvres de Cicéron? Et pourquoi sont-elles mises en relation avec les œuvres citées des autres auteurs?

On sait, par exemple, qu'il avait une grande admiration pour le De finibus, qui était l'une de ses premières acquisitions dans les années 1320 et qu'il connaissait en profondeur. Or ce texte n'est pas cité dans la lettre. Quelle est donc la signification du choix opéré par Pétrarque? La réponse est peut-être à rechercher, du moins en partie, dans la préface du livre II du De diuinatione, dans laquelle Cicéron passe en revue l'ensemble de ses œuvres philosophiques au sein du vaste programme qu'il s'est fixé et qui est de permettre au grand public d'étudier les principales questions philosophiques soulevées par les Grecs dans des œuvres latines. Or Cicéron classe ces œuvres en trois groupes: 1) l'Hortensius, exhortation à la philosophie en général et les Académiques, exposé de la doctrine à laquelle il adhère personnellement ; 2) le De finibus, étude du souverain bien et du souverain mal, et les Tusculanes; 3) le De natura Deorum, le De diuinatione, le De fato, consacrés à la religion ${ }^{16}$. À l'époque de cette préface, ni le De officiis ni le Cato maior ne sont écrits.

Ainsi, chaque œuvre de Cicéron citée dans la lettre 18 s'y trouverait en tant qu'emblème d'un aspect particulier de la pensée cicéronienne que l'on retrouve dans les trois premiers livres des Familières: les Académiques postérieures - qui relatent une discussion essentiellement historique pour savoir s'il convient de ranger Platon parmi les sceptiques ou les dogmatiques - représentent le premier groupe d'écrits

16 Voir Cicéron. 1970, Tusculanes, Paris, Belles Lettres, introduction, p. I-II. 
philosophiques, celui qui porte sur les bonnes raisons d'étudier la philosophie et surtout celle de Platon : en d'autres termes, il s'agit de la défense de la philosophie en tant que discipline théorique. Les Tusculanes symbolisent à elles seules le deuxième groupe, celui où Cicéron aborde la question du souverain bien et du souverain mal; elles sont préférées au Definibus, qui traite le sujet théoriquement, parce qu'elles vérifient dans l'ordre pratique la conclusion à laquelle aboutit le De finibus en plaçant le sage en situation (devant la mort, la douleur, le chagrin, les autres maux) : il s'agit de la défense de la philosophie, cette fois-ci plutôt en tant que discipline pratique ${ }^{17}$. Dans le contexte chrétien de l'époque de Pétrarque, les œuvres philosophiques de Cicéron concernant la religion païenne pouvant difficilement être prises en compte, Pétrarque les remplace par deux œuvres qui ne sont pas encore rédigées à l'époque du Dediuinatione, à savoir le De officiis et le Cato maior. Le premier tente de définir la morale pratique du uir bonus; le second, quant à lui, par l'entremise du vieux Caton, s'intéresse à ce que peuvent apporter la vieillesse et le passage du temps à la sagesse. En les faisant apparaître ici, Pétrarque aborde deux thèmes fondamentaux de sa correspondance, les modalités de l'engagement politique de l'humaniste ainsi que sa réflexion permanente face à la fuite du temps.

De l'ensemble du corpus philosophique légué par Cicéron, Pétrarque retient donc quatre œuvres présentées comme emblématiques, qui résument les quatre pôles d'interrogations pressantes soulevées, non seulement dans le livre III mais aussi

\footnotetext{
17 Voir Pétrarque, Fam. XVII, 1, 9-10, où le Cicéron des Tusculanes est loué pour avoir écrit, notamment, que « la philosophie n'est pas l'art des mots mais celui de la vie »; voir également, Fam. XVIII, 14, lettre entièrement consacrée au commentaire des Tusculanes.
} 
dans les deux premiers livres des Familières : les conditions de la connaissance (thème récurrent dans les trois livres), l'engagement politique (thème plus spécifique au livre III), la lutte contre les passions (thèmes récurrents dans les trois livres) et un antidote contre le passage du temps (thèmes propres aux livres I et II). Il n'est peut-être pas injustifié de les considérer comme une première base bibliographique.

Quant aux auteurs et aux œuvres qui sont mis en rapport avec ces textes de Cicéron et qui peuvent susciter l'étonnement, leur présence s'explique peut-être par le desiderium dont parle Pétrarque, mais aussi par la volonté de l'auteur de mettre diverses disciplines littéraires (l'érudition, l'épopée, la comédie, l'histoire) en relation avec la philosophie. Deux raisons peuvent être avancées: l'esprit humaniste considère la philosophie comme la discipline mère à partir de laquelle se déploient les autres disciplines et il présente en même temps comme une exigence intellectuelle la mise en relation des différentes disciplines à partir de la philosophie.

Si l'on s'intéresse maintenant au deuxième groupe, là encore, deux remarques s'imposent : à première vue, Pétrarque nous donne une liste totalement décousue; d'autre part, il mélange sans distinction les auteurs importants et secondaires, tel cet Hégésias de Cyrène placé entre Platon, Cicéron et Sénèque, comme si, par cette présentation, Pétrarque refusait d'introduire un canon d'auteurs importants qui pourrait nuire à la curiosité que doivent inspirer des auteurs mineurs.

Ce qui ressort, en tout cas, dans ce groupe, c'est l'importance du monde grec, notamment de la figure de Platon, qui est évoquée à travers deux œuvres, le Timée (ou la création du monde) et le Phédon (ou l'immortalité de l'âme). Certes, il 
n'est rien d'étonnant à ce que Pétrarque évoque ces deux œuvres, qui étaient les seules de Platon qu'il ait vraiment lues en latin ${ }^{18}$. L'intérêt est ailleurs, à savoir dans la mise en relation de ces deux œuvres avec d'autres.

Par exemple, le Timée lui évoque le nom de Solon'19. Si l'on songe à l'objet du Timée, à savoir la théorie platonicienne de la création du monde, qui fait de cette œuvre l'une des plus abstraites et des plus didactiques du philosophe athénien, il apparaît que, par sa mise en relation avec « le génie de Solon », c'est le rapport entre philosophie théorique et philosophie pratique qui intéresse Pétrarque. Cette remarque est confirmée par deux faits : dans la lettre 12 du livre III, Platon apparaît aux côtés du De officiis de Cicéron ${ }^{20}$, comme s'il fallait discrètement associer Platon, Cicéron et Solon autour de la vie publique et politique, dès lors tenter de faire coexister, sinon d'accorder, philosophie théorique et philosophie pratique. Dans une autre, Pétrarque écrit que « le prince des philosophes, Platon, réussit à se concilier l'affection de Denys, le tyran de Syracuse „21. Là encore, il s'agit d'orienter la lecture de Platon dans une direction morale et politique, ou plutôt d'orienter la réflexion vers le débat qui oppose philosophie théorique et philosophie pratique et de chercher à en opérer la synthèse. Il paraît clair, en tout cas, que la réflexion qui parcourt non seulement le

\footnotetext{
18 Le Timée était connu, au Moyen Âge, dans la version de Calcidius. Pour la connaissance du Phédon, voir Nolhac, 1907, II, p. 140 et Billanovich, 1961, «Petrarca e i classici», Petrarca e il petrarchismo, Bologna, Studi Petrarcheschi, 7, p. 26. Il est maintenant avéré que Pétrarque avait lu en latin également le Phédon. Voir Minio-Paluello, 1949 ; Ead. 1950.

${ }^{19}$ Nolhac, II, 140-142 et Pétrarque, Rer. Mem. I 36, 5.

20 Voir Pétrarque, Fam. III, 12, 6, citant librement Cicéron, Off. I, 7, 22, qui citait lui-même sommairement Platon, Epist. 9, 358 A.

21 Ibid. III, 22, 3.
} 
livre III, mais aussi les livres précédents - où alternent des lettres concernant l'engagement dans la vie publique et des lettres évoquant la tentation de la vie retirée du monde et consacrée à la méditation théorique ${ }^{22}$ - se trouve nourrie par une lecture de Platon confrontée à d'autres lectures, moins théoriques et plus engagées politiquement.

De la même manière, le Phédon de Platon, associé à Caton d'Utique et à Hégésias de Cyrène, sert de fondement bibliographique à la réflexion de Pétrarque sur l'immortalité de l'âme et sur le suicide que l'on trouve dans la lettre 13 du livre III. Plus largement, c'est la lecture du livre III qui permet de comprendre le rapprochement entre les trois noms : Caton est cité à plusieurs reprises dans ce livre, et toujours à l'occasion d'une méditation sur la mort ${ }^{23}$; quant à Hégésias de Cyrène, qui était surnommé l'avocat de la mort, Pétrarque a appris son existence par les Tusculanes de Cicéron ${ }^{24}$. Une fois de plus, on en revient au face à face Platon/Cicéron.

À la lumière de ces exemples, la démarche de Pétrarque commence à se dessiner : certes, il aime à rendre compte de toutes les sources, grecques et latines, explicites et implicites, à sa disposition sur un sujet, mais il cherche avant tout à offrir à son lecteur une confrontation de ces sources qui les fasse dialoguer entre elles, dans le but de résoudre les apparentes

\footnotetext{
22 Voir Fam. III, 3 et 4 - qui sont des lettres politiques concernant la prise de position de Pétrarque pour les Orsini - et Fam. III, 5 - dans laquelle Pétrarque traite des bienfaits de la solitude et qui préfigure le De Vita solitaria.

23 Voir Fam. III, 3, 8 ; la lettre III, 10 aborde le problème crucial du suicide.

24 Sur Hégésias de Cyrène, surnommé l'avocat de la mort, voir Dotti, 2002, p. 459. Sur les sources antiques liées à la problématique du suicide abordée par Pétrarque dans la lettre III, 10, voir Courcelle, 1943, p. 25 et suivantes (dans le chapitre consacré à Macrobe).
} 
apories. La bibliographie, telle que la conçoit Pétrarque, n'est pas seulement une liste exhaustive et récapitulative; elle est aussi le compte rendu de l'élaboration d'une réflexion concernant l'œuvre au sein de laquelle elle prend place. C'est d'ailleurs ce que confirme la suite du passage. Dans la même phrase, en effet, et sans transition, Pétrarque évoque les lettres de Cicéron: «je me suis fié à Sénèque avant de me fier à mes propres yeux »25 (Fam. III, 18, 5). On peut s'interroger sur les raisons de cette intrusion abrupte du genre épistolaire juste après les références philosophiques cicéronienne et platonicienne, que nous venons d'examiner. Il s'agit en fait d'une remarque sur le projet littéraire même de Pétrarque qui, à cet endroit précis, rappelle à son lecteur la double revendication du recueil des Familières, qui est d'être à la fois une correspondance et une œuvre philosophique. Si l'on se souvient du fait que le projet littéraire des Familières n'a vu le jour que grâce à la fusion des deux correspondances, sénéquienne et cicéronienne, celles-ci évoquées à ce moment-là de la lettre apparaissent comme le second versant de la base bibliographique des Familières.

Le groupe suivant peut paraître moins cohérent encore que les deux premiers sur la philosophie, l'auteur faisant cohabiter dans la même phrase Augustin, Servius, Lactance, Suétone, Aulu-Gelle et Favorinus. Une fois de plus, ce passage ne s'éclaire que s'il est mis en relation avec l'ensemble du livre III, voire avec les deux premiers livres des Familières. Pétrarque fait ici allusion au deuxième aspect de son projet littéraire qui, en quelque sorte, découle du premier et qui est traité dès la

25 [...] et de Ciceronis epystolis Senece priusquam oculis meis credidi. 
« lettre-proème $»^{26}:$ poser les bases d'une nouvelle théorie de la connaissance qui implique la reconnaissance de l'apport antique, suppose nécessairement une réflexion sur la compatibilité de la littérature chrétienne et de la littérature païenne et, par voie de conséquence, sur la place laissée à l'histoire dans cette tradition. L'humaniste indique ici quels sont les auteurs qui l'ont aidé à poser avec clarté, sinon à résoudre cette question récurrente de son œuvre. Ainsi, placer le païen Servius entre les chrétiens Augustin et Lactance n'est qu'une manière ramassée et directe de rappeler l'imbrication des mondes païen et chrétien. On peut remarquer qu'Augustin et Lactance eux-mêmes ont été les premiers à ouvrir la voie, puisque l'un n'hésite pas à parler de Sénèque et l'autre de Cicéron ${ }^{27}$. Là encore, le projet programmatique (la nécessité de faire dialoguer littératures païenne et chrétienne) tel que l'illustre la lettre-proème s'appuie sur une tradition que Pétrarque rappelle clairement dans cette lettre III, 18.

La nouveauté, voire l'originalité du nouveau programme humaniste mis au point par Pétrarque réside ailleurs. Tout d'abord dans le nom de Servius, qui est une source bibliographique importante pour l'humaniste 28 et qui est placé dans la lettre à plusieurs titres : en tant que commentateur de Virgile (Pétrarque incitant le futur humaniste à lire les auteurs sans oublier leurs commentateurs) ; en tant que symbole d'une pratique philologique qui a fleuri au IVe siècle et qu'il est urgent

\footnotetext{
26 Ibid. I, 1. Voir Dotti, 2002, p. 8.

27 Voir Pétrarque, Fam. XVII, 1, où il est question d'un fragment du De Republica de Cicéron, cité à partir de Lactance.

28 Dans les Familières, il n'apparaît qu'à deux reprises avant la lettre III, 18 : voir Fam. II, 3, 1 et Fam. III, 1, 11. Mais c'est à partir de Servius que Pétarque annota son Virgile. Sur l'importance de Servius aux yeux de Pétrarque, voir Mann, 2004.
} 
de restaurer; mais surtout, en tant que symbole du nouvel intérêt humaniste pour la découverte fébrile d'auteurs et de livres encore inaccessibles, notamment ceux de la littérature grecque, puisque Servius est accolé aux Argonautiques d'Apollonios, c'est-à-dire à un texte épique grec inconnu de Pétrarque $^{29}$. De même que la tradition chrétienne est indissociable de la tradition païenne, de même la culture latine est indissociable de la culture grecque ${ }^{30}$.

La seconde originalité de Pétrarque est d'accorder à l'histoire une place prépondérante au sein de ces nouvelles études ${ }^{31}$. Dès lors que, dans le nouveau programme, la tradition chrétienne n'est plus suffisante pour rendre compte du passé et que s'impose la lecture des grands maîtres de l'Antiquité, la place est libre pour une nouvelle discipline, qui naît en marge du christianisme, ou plutôt à partir de la confrontation féconde des mondes chrétien et païen. Ainsi s'expliquent les noms de Suétone et de Pline (associé à une Histoire romaine), d'AuluGelle (que Pétrarque rangeait parmi les historiens), de Favorinus, de Florus et de Tite-Live, qui forment la base historiographique à laquelle il se réfère sans cesse, dans ses œuvres historiques mais aussi dans les Familières ${ }^{32}$. Outre une œuvre philosophique, la correspondance de Pétrarque se veut également un ouvrage d'histoire.

\footnotetext{
29 Voir Nolhac, 1907, I, p. 193.

30 Sur le rôle de la culture grecque dans la pensée de Pétrarque, voir Snell, 1963.

31 Sur la place de l'histoire dans la pensée de Pétrarque, voir Pétrarque, 1943, p. XI-CXLIII ; Id. 1951; Id., 1996; Dotti, 1978.

32 Sur l'importance d'Aulu-Gelle, de Favorinus, de Florus, de Tite-Live, voir Nolhac, 1907, le chapitre intitulé « Pétrarque et les historiens latins ».
} 
Continuant son parcours, Pétrarque aborde ensuite un troisième aspect de la construction du nouvel édifice culturel : le rôle de l'éloquence et de la poésie. Il n'est pas question, ici, de rappeler l'importance de ces dernières chez l'humaniste, mais plutôt de se concentrer sur ce qui concerne précisément notre sujet. Le passage peut être lu à différents niveaux: d'abord, comme bibliographie des nombreuses lettres qui abordent la question problématique de la gloire ${ }^{33}$; ainsi le thème de la gloire littéraire s'ancre-t-il dans une longue tradition que Pétrarque ne fait que suivre. Il peut être lu ensuite comme bibliographie d'un autre thème cher à Pétrarque : la mise en relation nécessaire de l'éloquence et de la poésie, qui n'est pas un fait personnel et arbitraire mais répond, là encore, à une longue histoire pour laquelle l'éloquence de Cicéron est complémentaire du génie poétique de Virgile. Enfin, le passage présente l'intérêt d'évoquer les Saturnales sans prononcer le nom de leur auteur, Macrobe, qui n'en devient que plus présent ${ }^{34}$. À l'instar de Servius, Macrobe fut un défenseur important de l'hellénisme païen. Ainsi, comme il l'a fait pour la philosophie et pour l'histoire, Pétrarque encourage également la connaissance du plus grand nombre d'auteurs grecs, quand il s'agit de l'éloquence et de la poésie. Dans cette lettre se dessine de plus en plus clairement le programme qui sera réalisé par les humanistes de la génération suivante, au tout début du

\footnotetext{
33 Voir, notamment, Pétrarque, Fam. I, 2 et 3 ; II, 9 et III, 11.

34 Sur Macrobe et l'hellénisme païen, voir Courcelle, 1943, p. 3-36. La présence des Saturnales, dans la lettre III, 18 peut s'expliquer par le fait qu'il s'agit du récit d'un banquet littéraire autour des lettres grecques. Ce qui intéresse Pétrarque, c'est l'érudition grecque de Macrobe, qui est capable de lire Plutarque dans le texte et dont la culture englobe aussi bien les Ennéades de Plotin que l'œuvre de Porphyre (commentateur du Timée !). Si l'on ajoute son intérêt pour la grammaire comparée du grec et du latin, Macrobe est, aux yeux de Pétrarque, une auctoritas de choix.
} 
Quattrocento. Le nom de Macrobe permet également la transition avec les auteurs du groupe suivant.

En effet, Pétrarque évoque un cinquième et dernier groupe d'auteurs : les érudits encyclopédistes, dont il rappelle l'importance à ses yeux. Là encore, on peut s'interroger sur les raisons qui ont conduit Pétrarque à évoquer précisément trois auteurs : Priscien, Pline l'Ancien et Nonius Marcellus.

Outre leur importance sur le plan philologique, il se peut que les noms de Priscien et de Nonius soient cités parce qu'ils tentèrent l'un et l'autre d'associer étroitement culture latine et culture grecque dans leur œuvre. Le grammairien Priscien de Césarée est lié à la tentative de renouveau de l'hellénisme à Rome au tournant du VIe siècle, qui échoua avec la mort de Boèce et de Symmaque. En l'évoquant, Pétrarque rappelle qu'il faut tenter de restaurer la culture grecque et que cela ne peut se faire sans enseigner de nouveau la langue grecque. Ce qui échoua à l'époque de Priscien (les progrès de l'hellénisme byzantin apparaissant comme une ingérence sournoise en Italie aux yeux de Théodoric) doit être de nouveau tenté à l'époque de Pétrarque ${ }^{35}$.

Il est probable que Nonius Marcellus $^{36}$, dont le bilinguisme a fait l'objet d'études récentes, ait joué un rôle semblable aux yeux de Pétrarque.

35 Sur l'œuvre et l'enseignement de Priscien de Césarée à l'époque de l'empereur Théodoric, voir Courcelle, 1943, p.307-311; voir également Passalcqua, 1987, p. 40, 48.

36 Sur le bilinguisme de Nonius, voir Zaffagno, 2003 ; sur les auteurs qui font autorité pour Nonius (avant tout Virgile, Varron, Plaute, Cicéron, Titinius, Sisenna, Lucrèce, et peut-être Salluste), voir Barbarino, 2003 ; voir, également, les Prolegomena Noniana I (2000) ; II (2003) ; III (2004) ; IV et V (2005). 
Quant à Pline l'Ancien, cité entre les deux précédents, il serait peut-être à mettre en rapport direct avec notre sujet : non seulement, en effet, comme les deux grammairiens précédents, il offre une passerelle entre la Grèce et Rome, mais surtout, il présente un intérêt supplémentaire par un aspect particulier que l'on rencontre dans son œuvre, à savoir l'élaboration d'index en tête de chaque livre, dans lesquels figurent à la fois le contenu du livre, sorte de table des matières, et la liste des sources, cette dernière n'étant pas sans rapport avec notre lettre ${ }^{37}$.

Il se peut que Pétrarque ait vu en Pline un modèle à double titre. D'une part, l'érudit romain justifie sa démarche et explique pourquoi il tient à guider son lecteur. La table des matières procure à ce dernier une méthode pour entrer dans l'œuvre, comme il est écrit explicitement dans la préface à Vespasien :

[...] j'ai joint à cette épître la table de chacun des livres et j'ai apporté le plus grand soin à t'épargner de devoir les lire. Par là tu rendras service aussi aux autres lecteurs: au lieu de parcourir tout l'ouvrage, chacun ne cherchera que ce qu'il désire et saura où le trouver. Le procédé a déjà été employé dans notre littérature, par Valerius Soranus, pour les livres qu'il a intitulés Epoptides (Initiées) ${ }^{38}$ (Pline, H. N. I, 33).

Par ailleurs, Pline explique pourquoi il donne ses sources:

[...] j'ai placé la liste de mes sources en tête de mes livres. Car c'est à mon avis un geste généreux et plein d'une noble délicatesse que de publier les noms de ceux à qui l'on doit le

\footnotetext{
37 Voir supra, note 9.

38 [...] huic epistulae subiunxi summaque cura, ne legendos eos haberes, operam dedi. Tu per hoc et aliis praestabis ne perlegant, sed, ut quisque desiderabit aliquid, id tantum quaerat et sciat quo loco inueniat. Hoc ante me fecit in litteris nostris Valerius Soranus in libris quos Epotidwn inscripsit.
} 
succès de sa tâche, au lieu de faire comme la plupart des auteurs que j'ai maniés ${ }^{39}$ (H. N. I, 21).

Or démonstration a été faite de toute l'ambiguïté qu'il y avait chez Pline à citer consciencieusement ses sources : la démarche est certes « un aveu de modestie et un hommage à ses prédécesseurs", mais elle "illustre aussi sa culture et vaut comme argument d'autorité ${ }^{40}$. Ce qui est valable pour Pline, l'est peut-être aussi pour Pétrarque : l'accumulation ordonnée des auctoritates est, dans cette lettre, une condition nécessaire à l'humaniste pour asseoir sa propre auctoritas auprès du lecteur et pour légitimer ses conceptions théoriques concernant la nouvelle appréhension du savoir et des études qu'il appelle de ses vœux.

Quoi qu'il en soit, l'érudition, le savoir encyclopédique se voient accorder une place, dans le programme culturel de Pétrarque, qui semble en contradiction avec l'argument de la lettre III, 1 citée plus haut. La contradiction n'est qu'apparente : dans la pensée de Pétrarque, il existe bien une place pour l'érudition, mais seulement après que les autres champs du savoir ont été assimilés et qu'elle ne risque plus de nous entraîner sur les chemins foisonnants et stériles que dénonçait la première lettre du livre III. Ainsi dans cette dix-huitième lettre, Pétrarque répond-il à la question implicite posée au tout début du livre III : comment aborder les connaissances et quelle

\footnotetext{
39 [...] in his uoluminibus auctorum nomina praetexui. Est enim benignum, ut arbitror, et plenum ingenui pudoris fateri per quos profeceris, non ut plerique ex iis, quos attigi, fecerunt.

40 Naas, 2002, p. 143 et 144. Cet aveu recouvre vraisemblablement un enjeu important pour Pline, puisque, comme l'écrit Naas, Pline ignore « délibérément ou non, un illustre précédent: Varron a également donné la liste des sources de ses Res Rusticae; mais elles sont intégrées au début du texte et ne figurent pas séparées en tête, comme dans l'HN».
} 
place accorder à l'érudition dans le nouveau programme culturel?

La liste qui nous est donnée ici, dans cette lettre 18 du troisième livre, n'est pas, en tout cas pas seulement, un inventaire de la bibliothèque de Pétrarque ; par ailleurs, elle est loin d'être un recensement exhaustif de toutes les sources présentes dans les Familières. Il ne faudrait pas pour autant y voir une énumération accidentelle, fruit du hasard ou de l'inspiration momentanée de l'auteur de la lettre. Bien au contraire, les auteurs et les œuvres y sont choisis en fonction de critères précis qui, une fois mis à jour, permettent de penser que nous avons affaire à une démarche qui s'apparente à celle de la bibliographie (cryptée certes, mais non moins réelle), et qui nous amène à réfléchir sur ce que Pétrarque entendait par bibliographie, à savoir, une classification à plusieurs entrées.

On repère assez vite, en effet, que certaines des œuvres antiques évoquées sont en relation avec les thèmes abordés dans le livre III et, plus largement, dans les deux précédents. On peut donc affirmer sans trop de risques que, à un premier niveau, Pétrarque nous offre un récapitulatif des sources qui ont permis l'élaboration des trois livres programmatiques des Familières.

Dans un second temps, l'ordre d'apparition des œuvres et des auteurs révèle un autre classement, que l'on pourrait résumer ainsi : 1) Quel choix peut-on faire entre philosophie théorique et philosophie pratique? Entre vie solitaire et vie active? 2) Il y a-t-il compatibilité entre monde chrétien et monde païen ? Quel est le rôle de l'histoire ? 3) Quelle est la place de l'éloquence et de la poésie? 4) Quelle est celle de l'érudition? 
Sur un plan strictement littéraire, ces interrogations contribuent à situer le projet qu'est cette correspondance d'un genre un peu particulier : d'abord, elle se veut fusion de deux correspondances antiques, celle de Cicéron et celle de Sénèque ; mais avant d'être une correspondance, elle est une œuvre philosophique qui englobe philosophie théorique et philosophie pratique ; elle est une œuvre d'histoire, où l'on trouvera une confrontation féconde des sources chrétiennes et des sources païennes; elle est une œuvre rhétorique; enfin, elle est une œuvre d'érudition. Bref, elle est une œuvre qui englobe plusieurs genres.

Sur un autre plan, moins strictement littéraire, les grandes questions soulevées par la liste des auteurs indiquent un progrès dans le cheminement intellectuel: le futur humaniste est appelé à se former au double contact de la philosophie théorique et pratique ; à s'imprégner ensuite des valeurs et des leçons de la littérature chrétienne qui doit être lue en parallèle avec la littérature païenne (de cette confrontation sortira une meilleure compréhension de l'histoire); il doit ensuite poursuivre sa formation avec l'éloquence et la poésie; enfin, le savoir encyclopédique et l'érudition font partie de cette culture, mais constituent la dernière étape dans la formation, quand sont convenablement posées les autres bases méthodiques du savoir, qui permettent de faire des choix.

Dans cette page, nous serions en présence d'une démarche bibliographique impliquant à la fois un contenu (les noms des œuvres et des auteurs cités comme sources des Familières) et une méthode : les sources sont classées selon des critères thématiques répondant aux thèmes de réflexion de 
l'œuvre, mais elles le sont aussi selon un ordre hiérarchique qui répond aux différentes étapes de la formation intellectuelle du futur humaniste. En cela, la bibliographie dépasserait le simple cadre de l'œuvre.

\section{Bibliographie}

BARbarino, G. 2003, «Il tema dell'auctoritas in Nonio Marcello », Prolegomena Noniana II, a cura di Ferruccio Bertini, Genova, p. 81-108.

Billanovich, Giuseppe. 1943, Introduzione a Petrarca: Rerum memorandarum libri, Firenze, Sansoni.

-. 1947, Petrarca letterato. I. Lo scrittoio del Petrarca, Roma, Edizioni di storia e di letteratura.

-. 1951, La tradizione del testo di Livio e le origini dell'Umanesimo. I. Tradizione e fortuna di Livio tra Medioevo e Umanesimo, Antenore, Padova.

-. 1961, «Petrarca e i classici», dans Petrarca e il petrarchismo, Bologna.

-. 1996, Petrarca e il primo umanesimo, Padova, Studi sul Petrarca, 25.

CourCElLE, Pierre. 1943, Les lettres grecques en Occident. De Macrobe à Cassiodore, Paris, E. de Boccard.

DotTI, Ugo. 1978, Petrarca e la scoperta della coscienza moderna, Milano, Feltrinelli. 
—. 2002, in Pétrarque, Lettres Familières, Paris, Les Belles Lettres, coll. "Les Classiques de l'humanisme», vol. 1, introduction, p. XIII-LXXXV.

FEO, M. 1973, « Petrarca e la formazione dell'uomo », I problemi della pedagogia, nn. 5/6.

MANN, Nicholas. 2004, "De la poétique à l'image», dans Pétrarque: les voyages de l'esprit, Grenoble, Jérôme Millon, p. 47-72.

Minio-Paluello, L. 1949, Il Fedone con note autografe del Petrarca, Parigi, Bibl. Naz., Cod. Lat. 6567 A-, «Acc. Naz. D. Lincei, Rend. D. Cl. Di sc. Mor., st. E filol. », S. VIII, 4, p. 107-113.

-.1950, Plato Latinus... II, Phaedo interprete Henrico Aristippo, ed. L. Minio-Paluello, London, XII.

NAAS, Valérie. 2002, Le projet encyclopédique de Pline l'Ancien, Rome, École française de Rome.

NolHaC, Pierre. de. 2004, Pétrarque et l'humanisme, Genève,

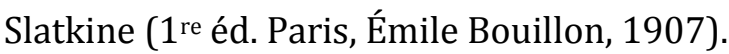

Passalcqua, M. 1987, Prisciani Caesariensis Opuscula, Roma, Sussidi eruditi.

PÉTRARQue, F.1943, Rerum Memorandarum libri, ed. critica a cura di G. Billanovich, Firenze, Sansoni.

—. 1999, De uita solitaria, éd. Ch. Carraud, Grenoble, Jérôme Millon.

—. 2002, Lettres Familières (introduction et notes de Ugo Dotti, traduction d'André Longpré), Paris, les Belles Lettres.

SABBADINI, Remigio. 1967, La scoperta dei codici latini e greci $n e^{\prime}$ secoli XIV e XV, a cura di E. Garin, Sansoni, Firenze, 2 vol. 
SNELL, Bruno. 1963, La cultura greca e le origini del pensiero europeo, trad. ital. Torino, Einaudi.

ZAFfagno, Elena. 2003, "Nonio e la sua lingua », Prolegomena Noniana II, a cura di Ferruccio Bertini, Genova, p. 63-74.

\title{
Résumé
}

Une lecture approfondie du texte et de ses références permet de lire l'histoire de la bibliographie dans une épître de Pétrarque (Lettres Familières, 3, 18). La lettre elle-même, une requête adressée à un ami censé lui trouver des livres, se présente avant tout comme un éloge de la littérature où l'humaniste semble procéder par association d'idées. Plus qu'à un inventaire de sa bibliothèque, nous avons affaire à une démarche qui s'apparente à celle de la bibliographie, à savoir une classification à plusieurs entrées. Pétrarque nous offre un récapitulatif des sources qui ont permis l'élaboration des trois livres programmatiques des Familières mais également un arrangement thématique de références philosophiques et littéraires. La démarche bibliographique de Pétrarque impliquerait ainsi à la fois un contenu et une méthode.

\begin{abstract}
A detailed analysis of the references and of the order of these references contained in a letter by Petrarch (Familiares, 3, 18) allows to recognize, within this text, a history of bibliography. The letter itself is a request, addressed to a friend who is supposed to provide the author with books; at first glance, the text is a praise of literature, composed on a loose association of ideas rather than by reason. But, there is more to be heard than a simple list of the books kept in Petrarch's library at the time of
\end{abstract}


the letter (after 1345). For the very intent of this list of books is bibliographical, as Petrarch proposes several possible classifications for a corpus of titles. He offers, in the first place, a summary of the sources he used for the three programmatic books of the Familiares. But he also proposes a thematic order for philosophical and literary readings. As such, this bibliographical project is both contents and methodology. 